\title{
Protection against inhaled oxidants through scavenging of oxidized lipids by macrophage receptors MARCO and SR-Al/II
}

\author{
Morten Dahl, ${ }^{1}$ Alison K. Bauer, ${ }^{2}$ Mohamed Arredouani, ${ }^{1}$ Raija Soininen, ${ }^{3}$ \\ Karl Tryggvason, ${ }^{4}$ Steven R. Kleeberger, ${ }^{2}$ and Lester Kobzik ${ }^{1}$

\begin{abstract}
1Department of Environmental Health, Harvard School of Public Health, Harvard Medical School, Boston, Massachusetts, USA. 2Laboratory of Respiratory Biology, National Institute of Environmental Health Sciences, NIH, Research Triangle Park, North Carolina, USA. ${ }^{3}$ Department of Medical Biochemistry and Molecular Biology, Biocenter Oulu, University of Oulu, Oulu, Finland. ${ }^{4}$ Division of Matrix Biology, Department of Medical Biochemistry and Biophysics, Karolinska Institutet, Stockholm, Sweden.
\end{abstract}

\begin{abstract}
Alveolar macrophages (AMs) express the class A scavenger receptors (SRAs) macrophage receptor with collagenous structure (MARCO) and scavenger receptor AI/II (SRA-I/II), which recognize oxidized lipids and provide innate defense against inhaled pathogens and particles. Increased MARCO expression in lungs of ozone-resistant mice suggested an additional role protecting against inhaled oxidants. After ozone exposure, $\mathrm{MARCO}^{-/-}$mice showed greater lung injury than did $\mathrm{MARCO}^{+/+}$mice. Ozone is known to generate oxidized, proinflammatory lipids in lung lining fluid, such as 5 $3,6 \beta$-epoxycholesterol ( $\beta$-epoxide) and 1-palmitoyl-2-(9'oxo-nonanoyl)-glycerophosphocholine (PON-GPC). Intratracheal instillation of either lipid caused substantial neutrophil influx in $\mathrm{MARCO}^{-/-}$mice, but had no effect in $\mathrm{MARCO}^{+/+}$mice. Normal AMs showed greater uptake in vitro of $\beta$-epoxide compared with $\mathrm{MARCO}^{-/-}$AMs, consistent with SRA function in binding oxidized lipids. SR-AI $/ \mathrm{II}^{-/}$- mice showed similar enhanced acute lung inflammation after $\beta$-epoxide or another inhaled oxidant (aerosolized leachate of residual oil fly ash). In contrast, subacute ozone exposure did not enhance inflammation in $\mathrm{SR}-\mathrm{AI} / \mathrm{II}^{-/-}$versus $\mathrm{SR}-\mathrm{AI} / \mathrm{II}^{+/+}$mice, reflecting increased AM expression of MARCO. These data identify what we believe to be a novel function for AM SRAs in decreasing pulmonary inflammation after oxidant inhalation by scavenging proinflammatory oxidized lipids from lung lining fluids.
\end{abstract}

\section{Introduction}

Inhaled oxidants are important causes of environmental lung injury, and the oxidative stress caused by air pollution and tobacco smoke can contribute to the pathogenesis of chronic obstructive pulmonary disease (COPD) and asthma $(1,2)$. The first point of contact between lung tissue and inhaled oxidants is the epithelial lining fluid. If levels of antioxidants in the lining fluid are inadequate to control activities of the inhaled oxidants, secondary oxidation products arise, which can pass on the oxidative stress to the surrounding milieu and underlying epithelium (3). By exposing bovine surfactant to ozone, Murphy and colleagues identified $5 \beta, 6 \beta$-epoxycholesterol ( $\beta$-epoxide) and 1-palmitoyl-2(9'-oxo-nonanoyl)-glycerophosphocholine (PON-GPC) as 2 major surfactant-derived oxidation products $(4,5)$. Both lipids are proinflammatory and may contribute to lung inflammation after ozone inhalation $(4,5)$. As surfactant covers a large fraction of the pulmonary epithelium, $\beta$-epoxide and PON-GPC can reach relatively high concentrations on the surface and be widely distributed during conditions of oxidative stress. Rapid clearance of oxidized surfactant lipids from the lung lining fluid therefore seems critical for a successful host response against inhaled oxidants.

Nonstandard abbreviations used: AM, alveolar macrophage; BAL, bronchoalveolar lavage; COPD, chronic obstructive pulmonary disease; $\beta$-epoxide, $5 \beta, 6 \beta$-epoxycholesterol; i.t., intratracheal(ly); MARCO, macrophage receptor with collagenous structure; MIP-2, macrophage inflammatory protein-2; PMN, polymorphonuclear leukocyte; PON-GPC, 1-palmitoyl-2-(9'-oxo-nonanoyl)-glycerophosphocholine; ROFA, residual oil fly ash; SRA, scavenger receptor class A; SR-AI/II, scavenger receptor AI/II. Conflict of interest: The authors have declared that no conflict of interest exists. Citation for this article: J. Clin. Invest. 117:757-764 (2007). doi:10.1172/JCI29968.
Macrophage receptor with collagenous structure (MARCO) and scavenger receptor $\mathrm{AI} / \mathrm{II}$ (SR-AI/II) are class A scavenger receptors (SRAs) on macrophages with potential functions in host defense against modified lipids (6). MARCO and SR-AI share the same overall domain structure, but differ in that MARCO has a longer extracellular domain and lacks an $\alpha$-helical coiled coil domain (7). SR-AI and SR-AII are 2 similar receptors generated through alternative splicing of a single gene. Macrophage scavenger receptors have long been known to clear modified lipids and contribute to foam cell formation during atherogenesis (8). They also function as part of innate defense systems in the lung, as scavenger receptors on alveolar macrophages (AMs) have been shown to bind environmental particles and bacteria (9-11). However, their role in protecting the lung against oxidized surfactant lipids generated by inhaled oxidants has not to our knowledge been examined previously.

Gene expression profiling identified increased MARCO expression in lungs of ozone-resistant mice, suggesting a protective role for this receptor. Using mice with genetic deletion of MARCO or SR-AI/II, we analyzed their specific in vivo roles in regulating lung inflammation in response to 2 inhaled oxidants, ozone and residual oil fly ash (ROFA). We also examined the in vivo role of MARCO and SR-AI/II in lungs exposed to oxidized surfactant lipids such as $\beta$-epoxide and PON-GPC. Absence of MARCO increased BAL markers of lung inflammation and injury after inhalation of ozone or ROFA leachate and after instillation of B-epoxie or PON-GPC. In vitro studies confirmed diminished uptake of oxidized lipids by MARCO-deficient AMs. Our data identify what we believe to be a novel role for MARCO 

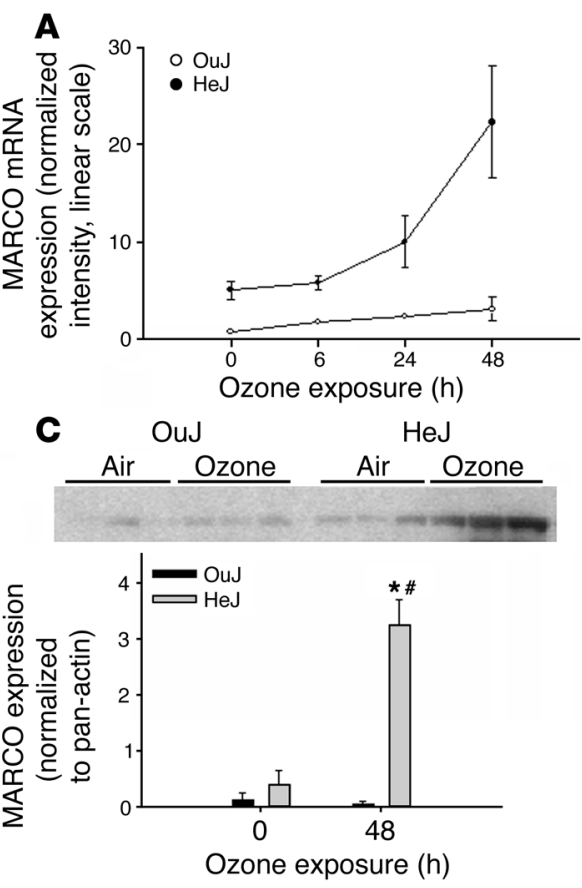

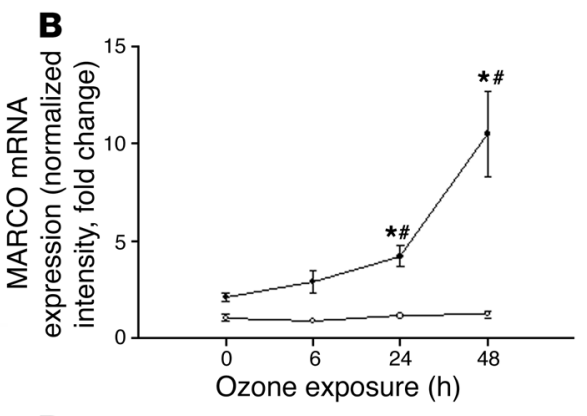

D

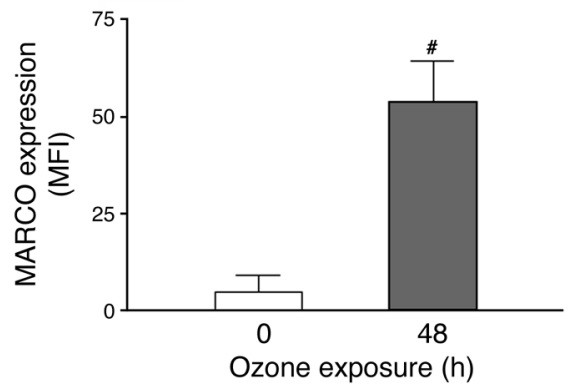

\section{Figure 1}

Ozone upregulates MARCO in lungs from ozone-resistant HeJ mice. HeJ or congenic ozone-sensitive OuJ mice were exposed to $0.3 \mathrm{ppm}$ ozone for up to 48 hours. Microarray analysis $(\mathbf{A})$ and RT-PCR (B) were performed on total RNA isolated from lung samples and showed increased MARCO mRNA expression in HeJ mice (filled symbols) compared with OuJ mice (open symbols). (C) Western blot analysis of lung tissue obtained after 48 hours of ozone exposure also showed increased MARCO protein expression. (D) Ozone upregulates MARCO on the surface of AMs of $\mathrm{C} 57 \mathrm{BL} / 6$ mice exposed to $0.3 \mathrm{ppm}$ ozone for 48 hours, as shown by increased fluorescence after flow cytometric analysis. Results shown are representative of 3 independent experiments. MFI, mean fluorescence intensity. ${ }^{\star} P<0.05$ versus ozone-treated OuJ; $\# P<0.05$ versus air-exposed control. and SR-AI/II in innate defenses against inhaled oxidants: beneficial scavenging of oxidized surfactant lipids from damaged lung lining fluids.

\section{Results}

Increased expression of MARCO after ozone exposure in vivo. In order to identify potential mediators of the ozone resistance previously observed in $\mathrm{C} 3 \mathrm{H} / \mathrm{HeJ}$ mice (12), we used microarray profiling and RT-PCR to analyze gene expression in lungs of ozone-resistant $(\mathrm{C} 3 \mathrm{H} / \mathrm{HeJ})$ and consomic, ozone-sensitive $(\mathrm{C} 3 \mathrm{H} / \mathrm{OuJ})$ mice. Analysis after exposure to $0.3 \mathrm{ppm}$ ozone for 48 hours showed increased MARCO mRNA expression at 24 and 48 hours in $\mathrm{C} 3 \mathrm{H} / \mathrm{HeJ}$ compared with $\mathrm{C} 3 \mathrm{H} / \mathrm{OuJ}$ mice (Figure 1, A and B). Western blot analysis of lung tissue from $\mathrm{C} 3 \mathrm{H} / \mathrm{HeJ}$ mice after 48 hours of $0.3-\mathrm{ppm}$ ozone exposure confirmed the increased MARCO expression at the protein level (Figure 1C).

Because the increased MARCO expression in whole-lung homogenate samples could be caused by increased lung macrophage numbers after ozone inhalation, we next isolated AMs from C57BL/ 6 mice also exposed to 0.3 ppm ozone for 48 hours and immunostained them for cell surface MARCO. We found that MARCO was significantly upregulated on the surface of AMs from $\mathrm{C} 57 \mathrm{BL} / 6$ mice in response to ozone exposure (Figure 1D), facilitating further analysis using C57BL/6 mice with a specific deletion of the MARCO gene $(9,13)$.

$M A R C O$ decreases inflammation in the lungs of mice exposed to ozone. To examine whether MARCO deficiency modulates lung inflammation in response to ozone, we exposed $\mathrm{MARCO}^{+/+}$and $\mathrm{MARCO}^{-/-}$ mice to $0.3 \mathrm{ppm}$ ozone for 48 hours and performed bronchoalveolar lavage (BAL). As expected, ozone caused increased neutrophil numbers in BAL fluid from $\mathrm{MARCO}^{+/+}$mice; however, the increase was 2-fold higher in $\mathrm{MARCO}^{-/-}$than in $\mathrm{MARCO}^{+/+}$mice $\left(20 \times 10^{4}\right.$ versus $8 \times 10^{4} ; P=0.003$; Figure $2 \mathrm{~A}$ ). Ozone mediates oxidative damage to the alveolocapillary membrane, and proteins leak from plasma into alveoli. Ozone caused increased BAL protein levels in
$\mathrm{MARCO}^{+/+}$mice, but the ozone-associated increase in BAL protein was slightly higher in $\mathrm{MARCO}^{-/-}$than $\mathrm{MARCO}^{+/+}$mice (Figure $2 \mathrm{~B}$ ). Ozone exposure also cause increased oxidative stress, which may result in the formation of 8-isoprostane from arachidonic acid phospholipids $(14,15)$. The relatively low concentration of ozone used in our exposures $(0.3 \mathrm{ppm})$ had no detectable effect on the level of 8-isoprostane in BAL samples from $\mathrm{MARCO}^{+/+}$mice, but increased BAL 8-isoprostane levels in $\mathrm{MARCO}^{-/-}$mice (Figure 2C). Hence, the presence of MARCO reduced the intensity of injury in lungs of $\mathrm{MARCO}^{+/+}$mice exposed to ozone, as measured by levels of neutrophils, protein, and 8-isoprostane.

MARCO decreases neutrophil accumulation in the lungs of mice exposed to aerosolized ROFA. To examine whether MARCO inhibits inflammation in lungs exposed to other inhaled oxidants besides ozone, we next exposed $\mathrm{MARCO}^{-/-}$mice to aerosolized leachate from ROFA, a surrogate for air pollution particulates $(100 \mathrm{mg} / \mathrm{ml}$ for 1 hour). The soluble metals present in dissolved ROFA (leachate) constitute most of the mass of ROFA samples and provide a useful model of acute lung injury following aerosol exposure (16). As expected, brief exposure to ROFA aerosol (1 hour) caused increased neutrophilia in $\mathrm{MARCO}^{+/+}$mice upon BAL analysis 18 hours later. Strikingly, the increase was 5-fold higher in $\mathrm{MARCO}^{-/-}$than in $\mathrm{MARCO}^{+/+}$mice $\left(5 \times 10^{4}\right.$ versus $1 \times 10^{4} ; P<0.001$; Figure $\left.2 \mathrm{D}\right)$. BAL isoprostane levels were minimal and not different between the 2 groups after these ROFA exposures which cause a lower absolute level of BAL neutrophilia than seen after ozone exposures (data not shown). After exposure to a higher concentration of ROFA (leachate of $300 \mathrm{mg} / \mathrm{ml}$ for 1.5 hours), $\mathrm{MARCO}^{-/-}$mice showed increased levels - similar to those following ozone exposure - of BAL polymorphonuclear leukocytes (PMNs) compared with $\mathrm{MARCO}^{+/+}$mice $\left(20 \times 10^{4}\right.$ versus $14 \times 10^{4}$, average of 5 mice per group) and a corresponding trend for increased BAL isoprostanes $(8.2 \mathrm{pg} / \mathrm{ml}$ versus $5.5 \mathrm{pg} / \mathrm{ml})$.

MARCO decreases inflammation in the lungs of mice exposed to oxidized lipids in vivo. Ozone exposure of lung lining fluid cholesterol creates $\beta$-epoxide, an oxidized surfactant lipid that is cytotoxic in vitro 

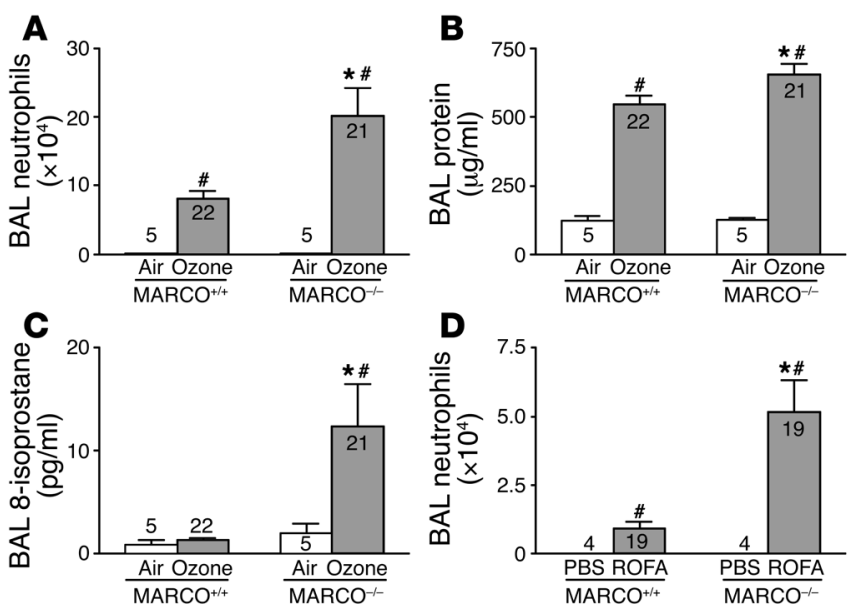

Figure 2

MARCO decreases inflammation in lungs of mice exposed to ozone and ROFA. (A-C) BAL samples obtained from MARCO-/- mice after exposure to $0.3 \mathrm{ppm}$ ozone for 48 hours showed higher levels of neutrophils (A), total protein (B), and 8-isoprostane (C) compared with controls. (D) BAL samples obtained from $\mathrm{MARCO}^{-/-}$mice 18 hours after exposure to aerosolized leachate from ROFA ( $100 \mathrm{mg} / \mathrm{ml}, 1$ hour) showed higher levels of neutrophils compared with controls. Number of mice per group is shown for each bar. ${ }^{*} P<0.05$ versus treated $\mathrm{MARCO}^{+/+}$group; ${ }^{*} P<0.05$ versus untreated.

and may contribute to lung inflammation after ozone inhalation $(4,17)$. Also, ozonolysis of unsaturated surfactant glycerophosphocholines creates PON-GPC (5). To determine whether MARCO protects against these oxidized surfactant lipids, we instilled $1 \mu \mathrm{g}$ $\beta$-epoxide or PON-GPC intratracheally (i.t.) into $\mathrm{MARCO}^{-/-}$mice. We found that i.t. instillation of either $\beta$-epoxide or PON-GPC into $\mathrm{MARCO}^{-/-}$mice caused substantial neutrophil influx, whereas these agents had no effect in $\mathrm{MARCO}^{+/+}$mice at this dose (Figure $3 \mathrm{~A}$ ). Instillation of a 100 -fold greater dose of either $\beta$-epoxide or PON-GPC into $\mathrm{MARCO}^{+/+}$mice did cause BAL neutrophilia $\left(2.3 \pm 2.8 \times 10^{4}\right.$ and $17 \pm 21 \times 10^{4}, n=5$ and 2 , respectively), confirming the proinflammatory potential of these agents when present in sufficient concentration. Instillation of $\beta$-epoxide and PON-GPC i.t. into $\mathrm{MARCO}^{-/-}$mice also caused increased levels of the neutrophil chemoattractant macrophage inflammatory pro- tein-2 (MIP-2) and total protein in BAL fluid, while no such effects were observed in $\mathrm{MARCO}^{+/+}$mice (Figure 3, B and C).

MARCO promotes lipid accumulation in AMs exposed to $\beta$-epoxide in vitro. To examine the role of MARCO in scavenging $\beta$-epoxide, we incubated $\mathrm{MARCO}^{-/-}$and $\mathrm{MARCO}^{+/+}$AMs with $\beta$-epoxide for 24 hours and quantitated cellular lipid content by flow cytometry after labeling with the fluorescent dye Nile Red. We found that $\mathrm{MARCO}^{-/-}$AMs exhibited diminished uptake compared with $\mathrm{MARCO}^{+/+} \mathrm{AMs}$, as evidenced by lower staining for cellular lipids after $\beta$-epoxide incubation (relative fluorescence, $\mathrm{MARCO}^{+/+}$, $30.8 \pm 10.7$; MARCO $^{-/-}, 10.3 \pm 10.3$ ). Viability of $\mathrm{MARCO}^{-/-}$and $\mathrm{MARCO}^{+/+}$AMs were similar at 95\% and 94\%, respectively, after 18 hours of $\beta$-epoxide incubation.

SR-AI/II decreases neutrophil accumulation in the lungs of mice exposed to ROFA and $\beta$-epoxide in vivo. In order to determine whether the other major macrophage SRA, SRA-I/II, also protects against inhaled oxidants, we exposed SR-AI/II ${ }^{+/+}$and SR-AI/II $/{ }^{-/}$mice to ROFA aerosols or $\beta$-epoxide by i.t. instillation and measured acute lung inflammatory responses by BAL analysis 7 hours later. While the ROFA aerosol caused a small increase in BAL neutrophils in $\mathrm{SR}-\mathrm{AI} / \mathrm{II}^{+/+}$mice, the increase was substantially higher in $\mathrm{SR}-\mathrm{AI} / \mathrm{II}^{-/-}$ mice $\left(9.9 \times 10^{4}\right.$ versus $0.8 \times 10^{4} ; P=0.002$; Figure $\left.4 \mathrm{~A}\right)$. Similarly, i.t. instillation of $\beta$-epoxide into SR-AI/II-/- mice caused substantial neutrophil influx, whereas it had no effect in $\mathrm{SR}-\mathrm{AI} / \mathrm{II}^{+/+}$mice $\left(11 \times 10^{4}\right.$ versus $0.2 \times 10^{4} ; P<0.001$; Figure 4B).

We also evaluated responses after the longer exposures to inhaled ozone ( $0.3 \mathrm{ppm}$ for 48 hours). In contrast to findings with $\mathrm{MARCO}^{-/-}$ mice, ozone caused similar small increases in neutrophils in SR-AI/II $\mathrm{II}^{+/+}$ and SR-AI/II $/$ - mice (Figure $4 \mathrm{C}$ ). Because ozone exposure was associated with increased expression of MARCO in normal or wildtype mice (Figure 1), we sought to determine whether increased expression of MARCO also occurs in SR-AI/II-/- mice. We isolated AMs from SR-AI $/ \mathrm{II}^{-/-}$mice exposed to $0.3 \mathrm{ppm}$ ozone for 48 hours, immunostained for cell surface MARCO, and quantified the expression using flow cytometry. We found that $\mathrm{SR}-\mathrm{AI} / \mathrm{II}^{-/-} \mathrm{AMs}$ exhibited substantially higher staining for MARCO after ozone exposure compared with air-exposed controls (Figure 5).

\section{Discussion}

Our interest in the role of the macrophage SRAs MARCO and SR-AI/II in regulating lung inflammation after ozone inhalation began with microarray data showing increased pulmonary
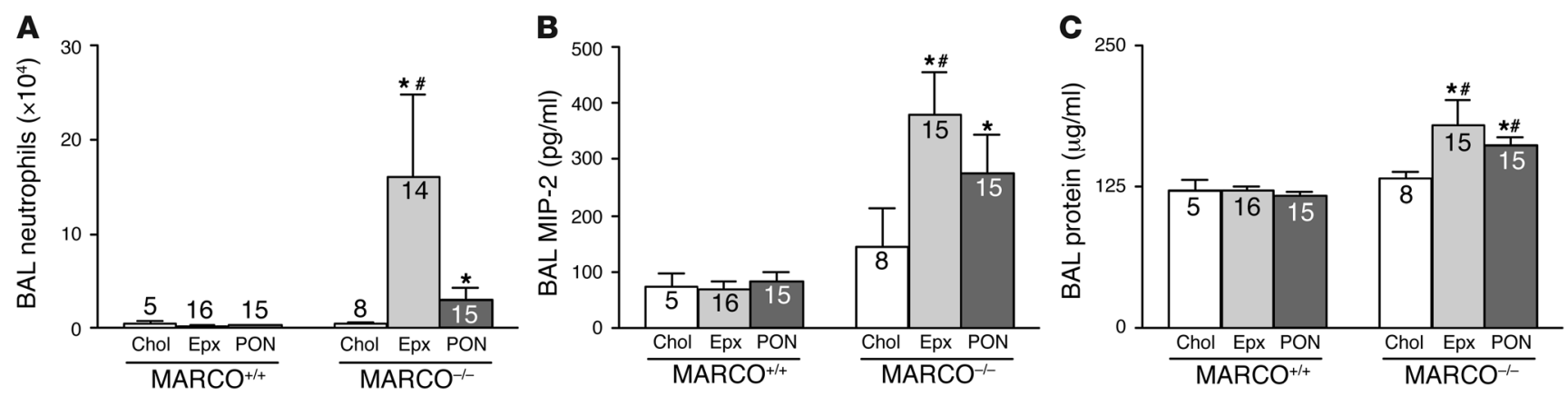

Figure 3

MARCO decreases inflammation in lungs of mice exposed to $\beta$-epoxide or PON-GPC i.t. BAL samples obtained from $\mathrm{MARCO}^{-/-}$mice 7 hours after i.t. instillation of $1 \mu \mathrm{g} \beta$-epoxide (Exp) or PON-GPC (PON) showed higher levels of neutrophils (A), MIP-2 (B), and total protein (C) compared with controls. Number of mice per group is shown for each bar. ${ }^{*} P<0.05$ versus respective treated $\mathrm{MARCO}^{+/+}$group; ${ }^{\#} P<0.05$ versus cholesterol (Chol) control. 

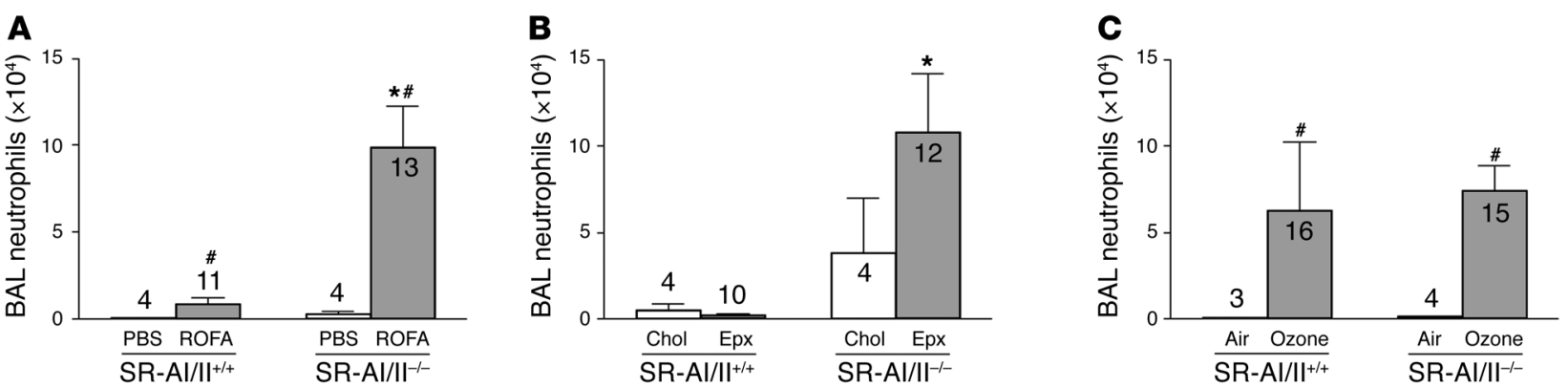

Figure 4

SR-AI/II decreases neutrophil accumulation in lungs of mice exposed to ROFA and i.t. $\beta$-epoxide, but not ozone. SR-Al//I-/- mice received aerosolized leachate from ROFA (100 mg/ml for 1 hour; $\mathbf{A}), 1 \mu \mathrm{g}$ i.t. $\beta$-epoxide (B), or $0.3 \mathrm{ppm}$ ozone (C) for 48 hours. Number of mice per group is shown for each bar. ${ }^{*} P<0.05$ versus treated SR-Al/II+/+ group; ${ }^{\#} P<0.05$ versus untreated control.

MARCO mRNA expression in ozone-resistant HeJ mice, but not in ozone-sensitive OuJ mice (Figure 1). While this initial hypothesis-generating data came from $\mathrm{HeJ}$ and $\mathrm{OuJ}$ mice, we subsequently determined that ozone inhalation also increased MARCO expression on AMs from $\mathrm{C} 57 \mathrm{BL} / 6$ mice, allowing more detailed analyses using mice deficient in MARCO (available on a C57BL/6 background). We found that absence of MARCO increased lung inflammation after inhalation of ozone and another inhaled oxidant, aerosolized ROFA leachate. These findings prompted further examination of the role of lung macrophage MARCO in uptake and removal of oxidized surfactant lipids such as $\beta$-epoxide and PON-GPC. Our analyses showed that MARCO mediated intracellular lipid accumulation after incubation of lung macrophages with $\beta$-epoxide in vitro. MARCO acted to protect lungs against inflammation after $\beta$-epoxide and PON-GPC instillation in vivo, as shown by increased inflammatory responses in $\mathrm{MARCO}^{-/-}$mice. Taken together, these data suggest what we believe to be a previously unrecognized role for lung macrophage SRAs in lung defense against inhaled oxidants through clearance of otherwise proinflammatory oxidized surfactant lipids from damaged lung lining fluids.

Our data show similar, but not identical, functions for the 2 receptors studied, MARCO and SR-AI/II. We found that SR-AI/II, also contributed to protection of the lungs against acute inflammation, as determined by the number of BAL neutrophils after ROFA and $\beta$-epoxide exposure. In contrast to $\mathrm{MARCO}^{-/-}$mice, however, SR-AI/II $/$ - mice did not show increased inflammation in experiments using 48 hours of exposure to ozone. After such ozone exposure, we observed increased expression of MARCO on AMs from both SR-AI/ $/ \mathrm{II}^{+/+}$and $\mathrm{SR}-\mathrm{AI} / \mathrm{II}^{-/-}$mice, suggesting a protective effect of MARCO in both groups. Moreover, it is noteworthy that ozone exposure did not cause increased expression of the gene encoding SR-AI/II in the same microarray analyses that revealed increased MARCO expression in the ozone-resistant HeJ mice (data not shown). One interpretation is that basal levels of SR-AI/II contribute to clearance of proinflammatory oxidized lipids generated by the acute challenges of ROFA aerosols or direct instillation of $\beta$-epoxide. Comparison of data from these more acute exposures with those of the 48-hour ozone experiments suggest that the basal and unchanged level of SR-AI/II is insufficient for optimal clearance of oxidized lipids generated during the longer exposure to ozone, a task mediated by the increased MARCO expression we observed in the ozone model. It is also possible that during repair of inflammation, scavenger receptors may be involved in binding and clearing cellular debris, thereby hampering further amplification of the inflammation in the lung. Hence, to the extent that such repair processes are initiated at the 48-hour time point of the ozone exposure, MARCO and SR-AI/II may limit inflammation through mechanisms beyond the proposed binding of oxidized lipids.

It is worth noting that ozone and ROFA generate different lipid oxidation products. Ozone produces specific lipid ozonation products plus nonspecific lipid autoxidation from both ozoneinitiated reactions within the epithelial lining fluid and after the onset of the inflammatory response. On the other hand, ROFA likely generates oxidized lipids via transition metal redox cycling as well as inflammation. Production of $\beta$-epoxide occurs in both scenarios, while PON-GCP should be relatively specific to ozone reactions. To facilitate comparison of ROFA to ozone exposures, we also analyzed BAL PMNs and isoprostanes using a higher ROFA concentration, which caused levels of PMNs comparable to those seen with ozone. Under these conditions, $\mathrm{MARCO}^{-/-}$mice showed a higher number of BAL PMNs and a trend toward higher 8-isoprostane levels. These data further support the conclusion that MARCO can scavenge a range of oxidatively modified lipids rather than being strictly specific for autoxidation-derived lipids.

When $\beta$-epoxide and PON-GCP were administered to $\mathrm{MARCO}^{+/+}$ mice, MARCO appeared to inhibit the inflammatory response to the oxidized lipids in full. When $\mathrm{MARCO}^{+/+}$mice were exposed to ROFA or ozone, MARCO only partly decreased the inflammatory

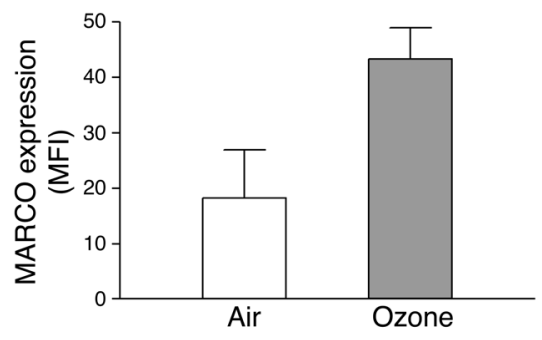

\section{Figure 5}

Ozone upregulates MARCO on the surface of SR-Al//I-- AMs. SR-Al/II-mice were exposed to $0.3 \mathrm{ppm}$ ozone for 48 hours, after which the AMs were isolated, labeled for MARCO, and analyzed by flow cytometry. Results shown are representative of 2 independent experiments. 
response, indicating that other oxidized products are capable of inducing inflammation in $\mathrm{MARCO}^{+/+}$mice. At 100 -fold greater doses, both $\beta$-epoxide and PON-GCP caused neutrophil influx in $\mathrm{MARCO}^{+/+}$mice 7 hours after instillation $\left(2.3 \times 10^{4}\right.$ and $17 \times 10^{4}$, respectively). At lower doses, the oxidized surfactant lipids did not seem critical to the inflammatory response in $\mathrm{MARCO}^{+/+}$mice, probably because MARCO and other scavenger receptors were capable of clearing the lipids sufficiently from the lung surface.

We have previously shown important functions for MARCO and $\mathrm{SR}-\mathrm{AI} / \mathrm{II}$ in innate immune responses against bacteria and environmental particles (9-11). The current data indicate that MARCO and SR-AI/II are also involved in clearing oxidized surfactant lipids in the lung. In atherosclerosis, scavenger receptors have long been known to take up oxidized lipids and contribute to foam cell formation $(8,18,19)$. While SR-AI/II is implicated in lipid loading of macrophage-derived foam cells during atherogenesis $(18,20-22)$, the receptor is also expressed on AMs, indicating that it may be engaged in metabolism of modified lipids in the lung. MARCO is upregulated on human foam cells (23) and on foam cells in atherosclerotic lesions from mice $(24,25)$, and a high-fat diet causes increased MARCO mRNA expression in the lungs and livers of C57BL/ 6 mice (25), further supporting a role for MARCO in lipid uptake in the lung.

Lipid ozonation products from surfactant are essential for the transmission of toxic signals to the pulmonary epithelium after ozone inhalation $(3,26,27)$. The oxidized surfactant lipids $\beta$-epoxide and PON-GPC are important lipid ozonation products implicated in this process. They are created from cholesterol and oleate- and palmitoyl-containing glycerophosphocholines, which constitute about $5 \%$ and $20 \%$, respectively, of the lung surfactant $(28,29)$. Also, $\beta$-epoxide is created from cholesterol in cell membranes. Both lipids are detected in vitro after exposing bovine surfactant to ozone $(4,5)$, and $\beta$-epoxide and nonanals are detected in vivo in BALs after exposing rodents to ozone $(17,30)$. We are not aware of previous studies measuring $\beta$-epoxide and PON-GPC using the 48-hour exposure conditions of the present study. However, exposing C57BL/6 mice to $0.5 \mathrm{ppm}$ ozone for 3 hours creates $2.5 \mathrm{ng} \beta$-epoxide per $\mathrm{ml} \mathrm{BAL}$, and unexposed lungs from $\mathrm{C} 57 \mathrm{BL} / 6$ mice have $\beta$-epoxide levels of approximately $95 \mathrm{ng}$ per lung (17). Our data using bolus instillation of $1 \mu \mathrm{g} \beta$-epoxide and PON-GPC provide a proof-of-principle, but future studies would benefit by more detailed measurement of the BAL levels of oxidized lipids during the 48-hour exposure to ozone.

Macrophages have previously been shown to take up $\beta$-epoxide (31), whereas PON-GPC uptake by macrophages is less well described. Lipid ozonation products regulate several proinflammatory cytokines, chemokines, and adhesion molecules (5, 32-37). Both $\beta$-epoxide and PON-GPC have specifically been associated with cytotoxic activities and expression of IL-8 $(5,32)$, an important neutrophil chemoattractant after ozone exposure $(3,38,39)$. In the present study, viability of AMs was relatively unaffected by $\beta$-epoxide incubation $(16 \mu \mathrm{g} / \mathrm{ml})$, in contrast to previous studies of cell lines in which this dose has been shown to have cytotoxic effects $(4,32,40)$. One explanation might be that the primary cells used in our assay resist the cytotoxic effect better than cell lines do. Alternatively, differences in our assay compared with previous studies may also account for the better cell survival we observed. Consistent with prior observations of $\beta$-epoxide and PON-GPC as potential stimulators of IL-8 release $(5,32)$, MIP-2, a rodent homolog of human IL-8, was markedly increased after i.t. instillation of $\beta$-epoxide or PON-GPC into $\mathrm{MARCO}^{-/-}$mice.
Both lung epithelial cells and macrophages express CXC chemokine mRNA and protein after ozone exposure in vivo $(38,41)$. MIP-2 expression after oxidant exposure has been localized immunohistochemically to both AMs and alveolar epithelial cells $(38,42)$. However, these data do not allow quantitation of the relative contribution of epithelial cells versus macrophages to MIP-2 release. One mechanism suggested by our present data is protection of lung epithelial cells from oxidized lipids by AM scavenging. Another possibility is that AM scavenger receptors divert oxidized lipids away from other, more proinflammatory receptors on the same cell and thereby reduce AM-derived MIP-2 and the inflammatory response that follows. In order to begin to address this question, we compared the capacity of AMs from $\mathrm{MARCO}^{+/+}$ and $\mathrm{MARCO}^{-/-}$mice to respond to oxidants by secretion of MIP-2. We observed similar fold increases in MIP-2 release in AMs exposed in vitro to $\mathrm{H}_{2} \mathrm{O}_{2}$ generated by a glucose oxidase-glucose system (43) (fold increase at $50 \mu \mathrm{g} / \mathrm{ml}$ glucose oxidase, $\mathrm{MARCO}^{+/+}$,

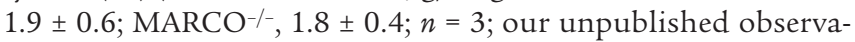
tions). These data suggest that $\mathrm{MARCO}^{-/-}$AMs retain the ability to respond to oxidants with proinflammatory cytokines, similar to previous observations using LPS (9). The question of the relative contribution of epithelial versus macrophage cytokine release when AM scavenger receptors are absent could be further analyzed in vitro. However, extrapolation to in vivo biology will remain difficult, as previous coculture experiments show enhanced release of CXC chemokines in response to environmental oxidants when AMs and lung epithelial cells are in contact (44).

Oxidants are present in air pollution and tobacco smoke or are released from activated leukocytes during lung inflammation. Increased oxidative stress in terms of increased BAL 8-isoprostane levels has been detected in lungs of smokers, patients with asthma, and patients with COPD (45). Therefore, we exposed $\mathrm{MARCO}^{-/-}$ and $\mathrm{MARCO}^{+/+}$mice to the smoke from 4 unfiltered cigarettes and after 6 hours found higher BAL neutrophils in $\mathrm{MARCO}^{-/-}$ than in $\mathrm{MARCO}^{+/+}$mice $\left(0.51\right.$ versus $0.07 \times 10^{4} ; n=8$ and $n=11$, respectively; $P=0.02$; our unpublished observations). These data indicate that MARCO protects the lung from acute cigarette smoke exposure. However, because cigarette smoke contains both particulate and gaseous proinflammatory components, whether MARCO protects by scavenging particles, smoke-generated oxidized lipids, or both, remains to be determined in future studies. When the lung lining fluid is under oxidative attack in injuries other than ozone-induced lung disease, significant concentrations of $\beta$-epoxide and PON-GPC may be produced and contribute to inflammation. In support of this idea, genetic studies show that the reduced activity of microsomal epoxide hydrolase, an enzyme that metabolizes $\beta$-epoxide (46), is associated with a higher risk of COPD and emphysema $(47,48)$. Other members of the epoxide hydrolase family besides microsomal epoxide hydrolase are also expressed in the lung (49-51), but their relation to the risk of lung disease is less well described.

In conclusion, our data indicate what we believe to be a previously unrecognized role for MARCO and SR-AI/II in innate defenses against inhaled oxidants, scavenging oxidized surfactant lipids from damaged lung lining fluids. This mechanism may apply to proinflammatory oxidized surfactant lipids other than the $\beta$-epoxide and PON-GPC we studied. We also speculate that MARCO and SR-AI/II could be involved in a general mechanism to dampen inflammation caused by the oxidized surfactant lipids generated during many forms of pulmonary inflammation and 
injury. Similar to the role for scavenger receptors in atherosclerosis $(8,52)$, SRAs internalize potentially proinflammatory oxidized lipids without engaging the typical phlogistic response in the lungs.

\section{Methods}

Animals. Mice (8-12 weeks old) genetically deficient in MARCO or SR-AI/ AII on the C57BL/ 6 background were used in all experiments but the initial gene expression profiling experiments, in which $\mathrm{C} 3 \mathrm{H} / \mathrm{OuJ}$ and $\mathrm{C} 3 \mathrm{H} / \mathrm{HeJ}$ mice were used. Age- and sex-matched $\mathrm{MARCO}^{+/+}$and $\mathrm{SR}-\mathrm{AI} / \mathrm{II}^{+/+}$ $\mathrm{C} 57 \mathrm{BL} / 6$ mice were used as controls. $\mathrm{MARCO}^{-/-}$mice were developed using homologous recombination and were backcrossed for at least 8 generations to the $\mathrm{C} 57 \mathrm{BL} / 6$ background $(9,13)$. SR-AI/II $/-\mathrm{C} 57 \mathrm{BL} / 6$ mice were generated by disrupting exon 4 of the gene encoding SR-AI/II (also known as macrophage scavenger receptor $1 ; \mathrm{Msr} 1$ ) as previously described (18) and were kindly provided by T. Kodama (University of Tokyo, Tokyo, Japan). All animals were housed in sterile microisolator cages and had no evidence of spontaneous infection. Ethical approval before all experimentation was obtained from the Harvard Medical Area Animal Use Committee, Boston, Massachusetts, USA.

Ozone exposure. Mice were placed in cages in a 145-1 stainless steel chamber with a Plexiglass door and then exposed to 0.3 ppm ozone for 48 hours. As a control, mice were exposed to atmospheric air in a neighboring chamber in the same room. During exposures, mice had continuous access to food and water. Ozone was generated by passing oxygen (Airgas East) through UV light, which was subsequently mixed with room air in the chamber. By the constant drawing of a sample of the chamber atmosphere through a sampling port, the ozone concentration within the chamber was monitored constantly by a UV photometric ozone analyzer (model 49; Thermo Electron Corp.), which was calibrated by a UV photometric ozone calibrator (model 49PS; Thermo Electron Corp.). In this ozone-induced lung disease model, there is acute lung inflammation with primary influx of neutrophils and production of proinflammatory mediators similar to ozone-induced lung injury in humans. BAL was performed within 1 hour after cessation of ozone exposure.

Aerosol exposure to ROFA leachate. A single sample (1 kg) of ROFA, obtained from the precipitator unit of a local power plant, was generously provided by J. Godleski (Harvard School of Public Health, Boston, Massachusetts, USA). ROFA was suspended at $100 \mathrm{mg} / \mathrm{ml}$ in PBS, pH 7.4, and sonicated for 10 minutes. After sitting for 30 minutes at room temperature, the ROFA suspension was incubated at $37^{\circ} \mathrm{C}$ with rotation for 4 hours and then centrifuged at $3,000 \mathrm{~g}$ for 10 minutes. The supernatant (leachate) was removed and used for aerosol exposures. Mice were exposed to a nebulized aerosol of ROFA leachate for 1 hour within individual compartments of a mouse "pie" chamber (Braintree Scientific) using a Pari IS2 nebulizer (SUN Medical Supply) connected to an air compressor (PulmoAID; DeVilbiss). BAL was performed 18 hours after the aerosol exposure, a time point previously determined to be most representative of maximal neutrophil accumulation and airway hyperresponsiveness in normal or Balb/C mice (16).

Lipid i.t. instillation. Mice were anesthetized by injecting 100-200 $\mu \mathrm{l}$ of $20 \mathrm{mg} / \mathrm{ml}$ ketamine hydrochloride (Fort Dodge Laboratories Inc.) intramuscularly into the right thigh. For each mouse, $50 \mu \mathrm{l} \mathrm{of} 20 \mu \mathrm{g} / \mathrm{ml} \beta$-epoxide (C2648; Sigma-Aldrich), $50 \mu \mathrm{l}$ of $20 \mu \mathrm{g} / \mathrm{ml}$ PON-GPC (870605; Avanti Polar Lipids), or $50 \mu \mathrm{l}$ of $20 \mu \mathrm{g} / \mathrm{ml}$ cholesterol (C8667; Sigma-Aldrich) was instilled i.t. BAL was performed 7 hours after i.t. lipid installation.

Affymetrix GeneChip array analysis. The left lungs of C3H/OuJ and C3H/HeJ mice exposed to $0.3 \mathrm{ppm}$ ozone or air ( $n=3$ per group) for 6 , 24 , or 48 hours of continuous exposure were processed for Affymetrix microarray analysis. Total RNA was isolated using TRIzol reagent (Invitrogen). cDNA was synthesized using SuperScript choice system (Invitrogen), followed by purification via phenol/chloroform extraction and labeling using an ENZO BioArray RNA transcript labeling kit. Purified biotin-labeled cRNA was then generated with QIAGEN's RNeasy kit and fragmented randomly to about $200 \mathrm{bp}$ (200 mM Tris-acetate, pH 8.2; $500 \mathrm{mM} \mathrm{KOAc;}$ and $150 \mathrm{mM} \mathrm{MgOAc}$ ). Each fragmented cRNA sample was hybridized to Affymetrix Murine Genome U74Av2 oligonucleotide arrays according to the manufacturer's instructions. Fluorescent images were read using a Hewlett-Packard G2500A Gene Array Scanner. Each GeneChip underwent a stringent quality-control regime using Microarray Analysis Software (version 5; Affymetrix) and the following parameters: cRNA fold changes (amount of cRNA obtained from starting RNA); scaling factor; percentage of "Present" calls; signal intensity; housekeeping genes; internal probe set controls; and visual inspection of the dat files for hybridization artifacts. The expression value (mean difference) for each gene was determined by calculating the mean of differences of intensity (perfect match intensity minus mismatch intensity) between its probe pairs. The expression analysis files created by Microarray Analysis Software (version 5) were transferred to GeneSpring 7.0 (Silicon Genetics) for statistical analyses and characterization of data. All samples were normalized in GeneSpring to $\mathrm{OuJ}$ air-exposed controls, similar to previous studies (53). After a stringent filtering of genes, including elimination of absent flags in all groups and inclusion of only those genes with significant interactions for strain and time $(P<0.05,2$-way ANOVA), we then identified changes in MARCO expression in $\mathrm{OuJ}$ versus $\mathrm{HeJ}$ mice at each time point.

RT-PCR. The same total RNA used for the microarray analysis $(1 \mu \mathrm{g})$ was reverse transcribed into cDNA in a volume of $50 \mu \mathrm{l}$. We then used $2.0 \mu \mathrm{l}$ of CDNA for quantitative PCR using a purchased probe set for MARCO according to the manufacturer's instructions (Mm00440265_m1; PerkinElmer). MARCO expression was normalized to 18S (Hs99999901_s1; PerkinElmer).

Western blotting. A piece of the right lung from the same mice as in the microarray analysis was homogenized in RIPA buffer, and supernatants containing total lung protein were obtained. Total protein $(100 \mu \mathrm{g})$ was denatured, fractionated by a $10 \%$ SDS-PAGE gel, and transferred onto PVDF membranes. After blocking with $5 \%$ nonfat dried milk in Tris-buffered saline, $\mathrm{pH} 7.4$, the membrane was incubated with rat anti-MARCO antibody (diluted 1:100; Cell Sciences). The membranes were then incubated with an anti-rat HRP-labeled secondary antibody (Santa Cruz Biotechnology Inc.), and protein signals were visualized with a chemiluminescent reagent (ECL Plus Western blotting detection system; Amersham Biosciences).

Cell isolation and BAL analyses. Mice were euthanized by an overdose of pentobarbital, and their lungs were lavaged 6 times with $0.8 \mathrm{ml}$ ice-cold PBS. Total cell yield was determined by hemocytometer, and differential counts were determined by microscopic evaluation of cytocentrifuge preparations stained with modified Wright-Giemsa. BAL protein levels were determined using the Bio-Rad protein assay, BAL 8-isoprostane was determined by competitive enzyme immunoassay (Cayman Chemical), and BAL MIP-2 was determined by ELISA assay (R\&D Systems) according to the manufacturers' instructions.

Flow cytometry. Lavaged AMs from nonexposed $\mathrm{MARCO}^{+/+}$and $\mathrm{MARCO}^{-/-}$ mice were plated at $160 \times 10^{3} /$ well in cell culture medium, $50 \mu \mathrm{g} / \mathrm{ml}$ cholesterol, or $16 \mu \mathrm{g} / \mathrm{ml} \beta$-epoxide in $1 \%$ heat-inactivated fetal bovine serum/penicillin/streptomycin/RPMI. After 24 hours, cells were washed 2 times in PBS, resuspended in $200 \mu \mathrm{l} \mathrm{PBS}$, and incubated on ice with $200-400 \mathrm{ng} / \mathrm{ml}$ Nile Red for 20-30 minutes. At least $90 \%$ of the cells were viable, as determined by Trypan Blue staining. After incubation, yellow-gold fluorescence emission was collected at $530-560 \mathrm{~nm}$ on a Coulter Epics Elite flow cytometer (Beckman Coulter). LPS level in the $\beta$-epoxide stock solution $(20 \mu \mathrm{g} / \mathrm{ml})$ was found to be below detection limit (less than $6.25 \mathrm{pg} / \mathrm{ml}$ ) using the Limulus amebocyte lysate assay (QCL-1000; BioWhittaker). 
Lavaged AMs from SR-AI/ $\mathrm{II}^{+/+}$and SR-AI/ $/ \mathrm{II}^{-/-}$mice exposed to air or $0.3 \mathrm{ppm}$ ozone for 48 hours were suspended at $2 \times 10^{6}$ per $\mathrm{ml}$ and incubated in $20 \mu \mathrm{g} / \mathrm{ml}$ rat anti-mouse CD16/CD32 (catalog no. 553140; BD Biosciences - Pharmingen) for 45 minutes on ice. After 45 minutes, $10 \mu \mathrm{g}$ FITC-conjugated rat anti-mouse MARCO antibody (catalog no. MCA1849FA; Serotec), $10 \mu \mathrm{g}$ FITC-conjugated rat anti-mouse IgG antibody (catalog no. MCA1211F; Serotec), or medium was added to each $400-\mu l$ cell suspension. After 55 minutes of incubation on ice, cells were washed 2 times in $1.2 \mathrm{ml} \mathrm{PBS}$ and resuspended in $5 \mu \mathrm{g} / \mathrm{ml}$ propidium iodine, $1 \%$ BSA, and PBS, after which fluorescence emission was collected at $530 \mathrm{~nm}$ on the flow cytometer using propidium iodine gating for live cells.

Statistics. Statistical analysis was performed with SPSS version 12. MannWhitney $U$ test or 2-tailed Student's $t$ test was used for comparisons of 2 groups. Results are reported as mean \pm SEM. A $P$ value less than 0.05 was considered significant.

1. MacNee, W., and Rahman, I. 2001. Is oxidative stress central to the pathogenesis of chronic obstructive pulmonary disease? Trends Mol. Med. 7:55-62.

2. Bowler, R.P. 2004. Oxidative stress in the pathogenesis of asthma. Curr. Allergy Asthma Rep. 4:116-122.

3. Mudway, I.S., and Kelly, F.J. 2000. Ozone and the lung: a sensitive issue. Mol. Aspects Med. 21:1-48.

4. Pulfer, M.K., and Murphy, R.C. 2004. Formation of biologically active oxysterols during ozonolysis of cholesterol present in lung surfactant. J. Biol. Chem. 279:26331-26338

5. Uhlson, C., et al. 2002. Oxidized phospholipids derived from ozone-treated lung surfactant extract reduce macrophage and epithelial cell viability. Chem. Res. Toxicol. 15:896-906.

6. Elomaa, O., et al. 1995. Cloning of a novel bacteriabinding receptor structurally related to scavenger receptors and expressed in a subset of macrophages. Cell. 80:603-609.

7. Arredouani, M.S., and Kobzik, L. 2004. The structure and function of marco, a macrophage class a scavenger receptor. Cell. Mol. Biol. (Noisy-le-grand). 50:OL657-OL665.

8. Murphy, J.E., Tedbury, P.R., Homer-Vanniasinkam, S., Walker, J.H., and Ponnambalam, S. 2005. Biochemistry and cell biology of mammalian scavenger receptors. Atherosclerosis. 182:1-15.

9. Arredouani, M., et al. 2004. The scavenger receptor MARCO is required for lung defense against pneumococcal pneumonia and inhaled particles. J. Exp. Med. 200:267-272.

10. Arredouani, M.S., et al. 2005. MARCO is the major binding receptor for unopsonized particles and bacteria on human alveolar macrophages. J. Immunol. 175:6058-6064.

11. Arredouani, M.S., et al. 2006. The macrophage scavenger receptor SR-AI/II and lung defense against pneumococci and particles. Am. J. Respir. Cell Mol. Biol. 35:474-478.

12. Kleeberger, S.R., Reddy, S., Zhang, L.Y., and Jedlicka, A.E. 2000. Genetic susceptibility to ozone-induced lung hyperpermeability: role of toll-like receptor 4. Am. J. Respir. Cell Mol. Biol. 22:620-627.

13. Mukhopadhyay, S., et al. 2006. MARCO, an innate activation marker of macrophages, is a class A scavenger receptor for Neisseria meningitidis. Eur. J. Immunol. 36:940-949.

14. Montuschi, P., Nightingale, J.A., Kharitonov, S.A., and Barnes, P.J. 2002. Ozone-induced increase in exhaled 8-isoprostane in healthy subjects is resistant to inhaled budesonide. Free Radic. Biol. Med. 33:1403-1408.

15. Long, N.C., et al. 2001. Ozone causes lipid peroxidation but little antioxidant depletion in exercising and nonexercising hamsters. J. Appl. Physiol. 91:1694-1700.

16. Hamada, K., Goldsmith, C.A., Suzaki, Y., Goldman,

\section{Acknowledgments}

This study was supported by NIH grants ES011008 and ES 00002 (to L. Kobzik) as well as by the Danish Medical Research Council (22-02-0549) to M. Dahl.

Received for publication August 4, 2006, and accepted in revised form December 12, 2006.

Address correspondence to: Lester Kobzik, Department of Environmental Health, Harvard School of Public Health, 665 Huntington Avenue, Boston, Massachusetts 02115, USA. Phone: (617) 432-2247; Fax: (617) 432-0014; E-mail: lkobzik@hsph. harvard.edu.

Morten Dahl and Alison K. Bauer contributed equally to this work.
A., and Kobzik, L. 2002. Airway hyperresponsiveness caused by aerosol exposure to residual oil fly ash leachate in mice. J. Toxicol. Environ. Health A. 65:1351-1365.

17. Pulfer, M.K., Taube, C., Gelfand, E., and Murphy, R.C. 2005. Ozone exposure in vivo and formation of biologically active oxysterols in the lung. J. Pharmacol. Exp. Ther. 312:256-264.

18. Suzuki, H., et al. 1997. A role for macrophage scavenger receptors in atherosclerosis and susceptibility to infection. Nature. 386:292-296.

19. Moore, K.J., et al. 2005. Loss of receptor-mediated lipid uptake via scavenger receptor A or CD36 pathways does not ameliorate atherosclerosis in hyperlipidemic mice. J. Clin. Invest. 115:2192-2201. doi:10.1172/JCI24061.

20. Babaev, V.R., Patel, M.B., Semenkovich, C.F., Fazio, S., and Linton, M.F. 2000. Macrophage lipoprotein lipase promotes foam cell formation and atherosclerosis in low density lipoprotein receptor-deficient mice. J. Biol. Chem. 275:26293-26299.

21. Takahashi, K., et al. 1992. Expression of macrophage scavenger receptors in various human tissues and atherosclerotic lesions. Clin. Biochem. 25:365-368.

22. Naito, M., Kodama, T., Matsumoto, A., Doi, T., and Takahashi, K. 1991. Tissue distribution, intracellular localization, and in vitro expression of bovine macrophage scavenger receptors. Am. J. Pathol. 139:1411-1423.

23. Tyson, K.L., Weissberg, P.L., and Shanahan, C.M. 2002. Heterogeneity of gene expression in human atheroma unmasked using cDNA representational difference analysis. Physiol. Genomics. 9:121-130.

24. Sakaguchi, H., et al. 1998. Role of macrophage scavenger receptors in diet-induced atherosclerosis in mice. Lab. Invest. 78:423-434.

25. Yoshimatsu, M., et al. 2004. Induction of macrophage scavenger receptor MARCO in nonalcoholic steatohepatitis indicates possible involvement of endotoxin in its pathogenic process. Int. J. Exp. Pathol. 85:335-343.

26. Pryor, W.A., Squadrito, G.L., and Friedman, M. 1995. A new mechanism for the toxicity of ozone. Toxicol. Lett. 82-83:287-293.

27. Connor, L.M., Ballinger, C.A., Albrecht, T.B., and Postlethwait, E.M. 2004. Interfacial phospholipids inhibit ozone-reactive absorption-mediated cytotoxicity in vitro. Am. J. Physiol. Lung Cell. Mol. Physiol. 286:L1169-L1178.

28. Notter, R.H. 2000. Lung surfactants: basic science and clinical applications. Marcel Dekker. New York, New York, USA. 130-176.

29. Postle, A.D., Heeley, E.L., and Wilton, D.C. 2001. A comparison of the molecular species compositions of mammalian lung surfactant phospholipids. Comp. Biochem. Physiol. A. Mol. Integr. Physiol. 129:65-73.

30. Pryor, W.A., Bermudez, E., Cueto, R., and Squadrito,
G.L. 1996. Detection of aldehydes in bronchoalveolar lavage of rats exposed to ozone. Fundam. Appl. Toxicol. 34:148-156.

31. Cao, J., Fales, H.M., and Schaffner, C.P. 1995. Cellular sterol accumulation stimulated by cholesterol 5 beta, 6 beta-epoxide in J774 macrophages. Proc. Soc. Exp. Biol. Med. 209:195-204.

32. Lemaire-Ewing, S., et al. 2005. Comparison of the cytotoxic, pro-oxidant and pro-inflammatory characteristics of different oxysterols. Cell Biol. Toxicol. 21:97-114.

33. Kafoury, R.M., et al. 1999. Induction of inflammatory mediators in human airway epithelial cells by lipid ozonation products. Am. J. Respir. Crit. Care Med. 160:1934-1942.

34. Kafoury, R.M., et al. 1998. Lipid ozonation products activate phospholipases A2, C, and D. Toxicol. Appl. Pharmacol. 150:338-349.

35. Leitinger, N., et al. 1999. Structurally similar oxidized phospholipids differentially regulate endothelial binding of monocytes and neutrophils. Proc. Natl. Acad. Sci. U.S.A. 96:12010-12015.

36. Yeh, M., et al. 2001. Increased transcription of IL-8 in endothelial cells is differentially regulated by TNF-alpha and oxidized phospholipids. Arterioscler. Thromb. Vasc. Biol. 21:1585-1591.

37. Leonarduzzi, G., et al. 2005. Oxysterol-induced upregulation of MCP-1 expression and synthesis in macrophage cells. Free Radic. Biol. Med. 39:1152-1161.

38. Chang, M.M., Wu, R., Plopper, C.G., and Hyde, D.M. 1998. IL-8 is one of the major chemokines produced by monkey airway epithelium after ozone-induced injury. Am. J. Physiol. 275:L524-L532.

39. Jaspers, I., Flescher, E., and Chen, L.C. 1997. Ozoneinduced IL-8 expression and transcription factor binding in respiratory epithelial cells. Am. J. Physiol. 272:L504-L511.

40. Ryan, L., O'Callaghan, Y.C., and O'Brien, N.M. 2004. Comparison of the apoptotic processes induced by the oxysterols 7beta-hydroxycholesterol and cholesterol-5beta,6beta-epoxide. Cell Biol. Toxicol. 20:313-323.

41. Driscoll, K.E., Simpson, L., Carter, J., Hassenbein, D., and Leikauf, G.D. 1993. Ozone inhalation stimulates expression of a neutrophil chemotactic protein, macrophage inflammatory protein 2 . Toxicol. Appl. Pharmacol. 119:306-309.

42. Deng, H., Mason, S.N., and Auten, R.L., Jr. 2000. Lung inflammation in hyperoxia can be prevented by antichemokine treatment in newborn rats. Am. J. Respir. Crit. Care Med. 162:2316-2323.

43. Ning, Y., et al. 2004. Particle-epithelial interaction: effect of priming and bystander neutrophils on interleukin-8 release. Am. J. Respir. Cell Mol. Biol. 30:744-750.

44. Tao, F., and Kobzik, L. 2002. Lung macrophageepithelial cell interactions amplify particle-medi- 
ated cytokine release. Am. J. Respir. Cell Mol. Biol. 26:499-505

45. Montuschi, P., Barnes, P.J., and Roberts, L.J., 2nd. 2004. Isoprostanes: markers and mediators of oxidative stress. FASEB J. 18:1791-1800.

46. Aringer, L., and Eneroth, P. 1974. Formation and metabolism in vitro of 5,6-epoxides of cholesterol and beta-sitosterol. J. Lipid Res. 15:389-398.

47. Smith, C.A., and Harrison, D.J. 1997. Association between polymorphism in gene for microsomal epoxide hydrolase and susceptibility to emphysema.
Lancet. 350:630-633.

48. Hersh, C.P., et al. 2006. Genetic association analysis of functional impairment in chronic obstructive pulmonary disease. Am. J. Respir. Crit. Care Med. 173:977-984.

49. Newman, J.W., Morisseau, C., and Hammock, B.D. 2005. Epoxide hydrolases: their roles and interactions with lipid metabolism. Prog. Lipid Res. 44:1-51. 50. Petruzzelli, S., et al. 1992. Cigarette smoke inhibits cytosolic but not microsomal epoxide hydrolase of human lung. Hum. Exp. Toxicol. 11:99-103.
51. Willey, J.C., et al. 1997. Quantitative RT-PCR measurement of cytochromes p450 1A1, 1B1, and 2B7, microsomal epoxide hydrolase, and NADPH oxidoreductase expression in lung cells of smokers and nonsmokers. Am. J. Respir. Cell Mol. Biol. 17:114-124. 52. Savill, J. 1998. Apoptosis. Phagocytic docking without shocking. Nature. 392:442-443.

53. Cho, H.Y., Reddy, S.P., Debiase, A., Yamamoto, M., and Kleeberger, S.R. 2005. Gene expression profiling of NRF2-mediated protection against oxidative injury. Free Radic. Biol. Med. 38:325-343. 